\title{
A precessing Be disk as a possible model for occultation events in GX 304-1
}

\author{
M. Kühnel, ${ }^{1 \star}$ R. E. Rothschild ${ }^{2}$ A. T. Okazaki, ${ }^{3}$ S. Müller, ${ }^{1}$ K. Pottschmidt, ${ }^{4,5}$ \\ R. Ballhausen, ${ }^{1}$ J. Choi, ${ }^{6}$ I. Kreykenbohm, ${ }^{1}$ F. Fürst, ${ }^{7}$ D.M. Marcu-Cheatham,,${ }^{4,5}$ \\ P. Hemphill, ${ }^{8}$ M. Sagredo, ${ }^{1}$ P. Kretschmar, ${ }^{7}$ S. Martínez-Núñez, ${ }^{9}$ J. M. Torrejón, ${ }^{10}$ \\ R. Staubert, ${ }^{11}$ and J. Wilms ${ }^{1}$ \\ ${ }^{1}$ Dr. Karl Remeis-Observatory \& ECAP, Universität Erlangen-Nürnberg, Sternwartstr. 7, 96049 Bamberg, Germany \\ ${ }^{2}$ Center for Astrophysics and Space Sciences, University of California, San Diego, La Jolla, CA 92093, USA \\ ${ }^{3}$ Faculty of Engineering, Hokkai-Gakuen University, Toyohira-ku, Sapporo 062-8605, Japan \\ ${ }^{4}$ CRESST/CSST/Department of Physics, UMBC, Baltimore, MD 21250, USA \\ ${ }^{5}$ NASA Goddard Space Flight Center, Greenbelt, MD 20771, USA \\ ${ }^{6}$ Harvard Smithsonian Center for Astrophysics, Cambridge, MA 02138, USA \\ ${ }^{7}$ European Space Astronomy Centre (ESA/ESAC), Operations Departement, 28691 Villanueva de la Cañada, Madrid, Spain \\ ${ }^{8}$ MIT Kavli Institute for Astrophysics and Space Research, 77 Massachusetts Ave, Cambridge, MA 02139, USA \\ ${ }^{9}$ Instituto de Física de Cantabria, Avda. los Castros s/n, 39005 Santander, Spain \\ ${ }^{10}$ Instituto Universitario de Física Aplicada a las Ciencias y las Tecnologías, University of Alicante, P.O. Box 99, 03690 Alicante, Spain \\ ${ }^{11}$ Institut für Astronomie und Astrophysik, Universität Tübingen, Sand 1, 72076 Tübingen, Germany
}

Accepted XXX. Received YYY; in original form ZZZ

\begin{abstract}
We report on the RXTE detection of a sudden increase in the absorption column density, $N_{\mathrm{H}}$, during the 2011 May outburst of GX 304-1. The $N_{\mathrm{H}}$ increased up to $\sim 16 \times 10^{22}$ atoms cm $^{-2}$, which is a factor of 3-4 larger than what is usually measured during the outbursts of GX 304-1 as covered by RXTE. Additionally, an increase in the variability of the hardness ratio as calculated from the energy resolved RXTE-PCA light curves is measured during this time range. We interpret these facts as an occultation event of the neutron star by material in the line of sight. Using a simple 3D model of an inclined and precessing Be disk around the Be type companion, we are able to qualitatively explain the $N_{\mathrm{H}}$ evolution over time. We are able to constrain the Be-disk density to be on the order of $10^{-11} \mathrm{~g} \mathrm{~cm}^{-3}$. Our model strengthens the idea of inclined Be disks as origin of double-peaked outbursts as the derived geometry allows accretion twice per orbit under certain conditions.
\end{abstract}

Key words: X-rays: binaries - stars: Be - stars: neutron - occultations - pulsars: individual: GX 304-1

\section{INTRODUCTION}

The $\mathrm{H} \alpha$ emission line observed in the optical spectra of many Bstars is believed to originate from a circumstellar disk (see, e.g., Hanuschik 1996). The $\mathrm{H} \alpha$ line profile in these stars often shows a double-peaked structure due to intrinsic rotation of the disk. These emission features are denoted with an " $e$ " in the stellar spectral type, which is why these stars are known as Be stars. The Be-star disks are supposed to form by the viscous diffusion of gas ejected from the central star (Lee et al. 1991). They are geometrically thin and rotating at Keplerian velocities (see Rivinius et al. 2013, for a recent review).

Circumstellar disks around main-sequence stars have been pro-

^ E-mail: matthias.kuehnel@sternwarte.uni-erlangen.de posed to explain the transient nature of some X-ray binaries (Rappaport et al. 1978). In these so-called Be X-ray binaries (BeXRBs) a compact object is on a wide and eccentric orbit around a Be-type companion star. Around the periastron passage mass transfer from the Be-star disk becomes possible, which results in a sudden and luminous X-ray outburst. Furthermore, the Be disk in BeXRBs is tidally truncated at a certain resonance radius due to the presence of the orbiting neutron star (Reig et al. 1997; Negueruela \& Okazaki 2001; Okazaki et al. 2002). This truncation leads to higher surface densities of BeXRB-disks compared to disks around isolated stars (Okazaki et al. 2002, in agreement with findings by Zamanov et al. 2001). However, Zamanov et al. (2001) have drawn this conclusion based on measurements of $\mathrm{H} \alpha$ equivalent widths and the peak separation of the line profile.

BeXRBs offer the opportunity to measure directly the Be- 
Table 1. Orbital parameters of GX 304-1 after Sugizaki et al. (2015, $\gamma$ free model).

$\begin{array}{ll}\text { Orbital period, } P_{\text {orb }}(\mathrm{d}) & =132.189 \pm 0.022 \\ \text { Projected semi-major axis, } a \sin i(\mathrm{lt}-\mathrm{s}) & =601 \pm 38 \\ \text { Eccentricity, } e & =0.462 \pm 0.019 \\ \text { Time of periastron passage, } \tau(\mathrm{MJD}) & =55425.6 \pm 0.5 \\ \text { Longitude of periastron, } \omega\left({ }^{\circ}\right) & =130.0 \pm 4.4\end{array}$

disk density independently of other energy ranges such as optical wavelengths. The particle density in the line of sight determines the absorption of X-rays at energies below $\sim 10 \mathrm{keV}$. As the X-ray line of sight is fixed to the neutron star, which moves along its orbit, the particle density around the Be star can be probed.

The BeXRB GX 304-1, detected in 1967 by a balloon experiment (Lewin et al. 1968), consists of a shell Be-star of type B2Vne (Mason et al. 1978; Parkes et al. 1980) or B0.7Ve (Liu et al. 2006) and a neutron star which shows X-ray pulsations around $\sim 272 \mathrm{~s}$ (McClintock et al. 1977). It is located at a distance of 2.4(5) kpc (Parkes et al. 1980) probably behind the Coalsack Nebula, a dark molecular cloud on the Southern sky at a distance of $\sim 150 \mathrm{pc}$ (see Nyman 2008, and references therein).

GX 304-1 often remained in states of quiescence as, e.g., between 1980 and 2008. On the other hand, it showed regular outbursts previously with intervals of 132.5(4) d as found by Priedhorsky \& Terrell (1983), who interpreted this periodicity as the orbital period of the system. This makes GX 304-1 a prime example of sources with a highly unpredictable outburst behaviour (Manousakis et al. 2008; Klochkov et al. 2012). The source again underwent regular outbursts in late 2009 until mid 2013. Recently, Sugizaki et al. (2015) confirmed the orbital period to be $P_{\text {orb }}=132.189(22) \mathrm{d}$ based on the outburst spacing in the MAXI-GSC light curves during this series. Together with pulse frequency measurements by Fermi-GBM (Finger et al. 2009) they derived the remaining orbital parameters of GX 304-1 (see Table 1).

In rare cases BeXRBs undergo so-called double-peaked outbursts, where two consecutive outbursts can be observed, which are not separated by the usually observed phase of quiescence. In order to explain the observed double-peaked outbursts of GX 304-1 in 2012, Postnov et al. (2015) proposed that the Be disk is probably inclined with respect to the orbital plane. This is in line with smoothed particle hydrodynamic (SPH) simulations performed by Okazaki et al. (2013), who concluded that the occurrence of socalled "giant" type II X-ray outbursts are probably triggered by a misaligned Be disk.

This paper is based on the spectral results of the accompanying paper by Rothschild et al. (2017, hereafter R17), who analysed 72 RXTE observations taken during four outbursts between March 2010 and May 2011. Here, we particularly focus on the evolution of the absorption column density, $N_{\mathrm{H}}$, over time. The paper is organized as follows: in Section 2 we briefly describe the data reduction process performed by R17. Section 3 gives a summary and discussion of their spectral results, which are then modelled by a simple 3D-model of a precessing Be disk in Sect. 4. We present our conclusions in Sect. 5 .

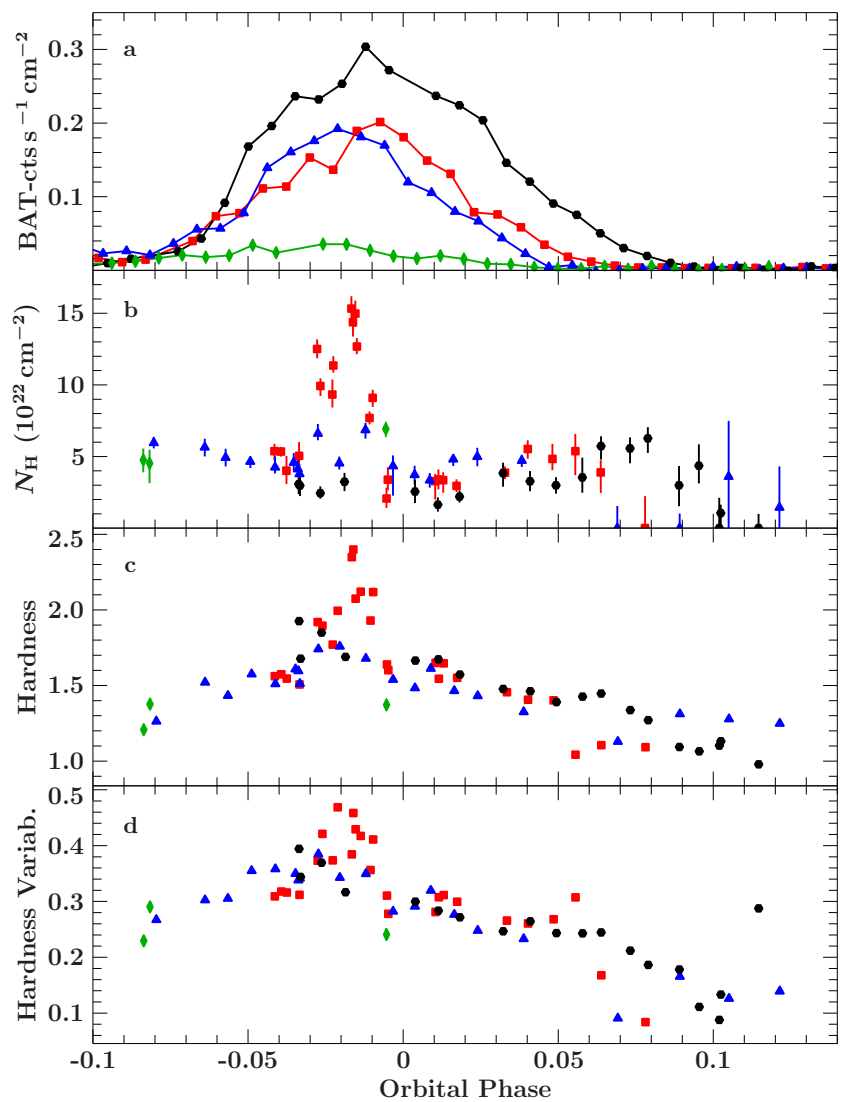

Figure 1. a) Count rate of GX 304-1 in Swift-BAT as a function of orbital phase during the outbursts in $2010 \mathrm{March} / \mathrm{April}$ (green diamonds), 2010 August (black circles), 2010 December (blue triangles), and 2011 May (red squares). b) Evolution of the absorption column density, $N_{\mathrm{H}}$, and c) evolution of the hardness ratio and $\mathbf{d}$ ) its variability over the orbital phase.

\section{OBSERVATIONS AND DATA REDUCTION}

\subsection{Observations}

RXTE has observed GX 304-1 during four outbursts in 2010 and 2011 (see Fig. 1a). In total, 72 individual observations are available with exposure times from a few $100 \mathrm{~s}$ up to $18 \mathrm{ks}$ each. The corresponding observation IDs and further basic informations about each observation can be found in R17, Tab. 1. The exposure times of three observations were less than the GX 304-1's pulse period of $\sim 272 \mathrm{~s}$ (McClintock et al. 1977). These observations have been excluded from the spectral analysis, which focuses on pulse phase averaged spectra.

\subsection{Data Reduction}

The data reduction process and the spectral results analysed further in Section 3 are the same as in R17. In the following a brief summary of the data reduction is given.

The Proportional Counter Array (PCA; Jahoda et al. 2006) onboard RXTE consisted of five identical Proportional Counter Units (PCU0-5). For spectral analysis only data from the top layer of PCU2 have been extracted since this PCU is known to be the best calibrated one (Jahoda et al. 2006). We used the tools distributed by HEASOFT v6.18 in order to extract spectra and lightcurves using the standard2f data mode. We avoided PCU2-events up to 30 
minutes from the start of the previous passage of the South Atlantic Anomaly and for elevation angles smaller than $10^{\circ}$ above the Earth's limb. Due to the high count rate of GX 304-1 during many of the 72 observations we used 0.5 as the highest accepted value for the electron ratio. For spectral analysis the energy range of 3-60 keV range has been used and no further spectral binning was applied. Due to the high quality of the PCA-spectra during a few observations, we had to add systematic uncertainties of $0.5 \%$ at channel energies $\leq 15 \mathrm{keV}$ and of $1.5 \%$ at higher energies in order to achieve a reduced $\chi^{2}, \chi_{\text {red }}^{2}$, near unity (see R17 for the affected ObsIDs). The PCU2 light curves were extracted with a $16 \mathrm{~s}$ time resolution in the detector channels 4-15 and 15-60 (corresponding to the energy bands $2.9-7.7 \mathrm{keV}$ and 7.7-30.0 keV, respectively). These channels have been chosen such that the ratio of the light curves, i.e., a hardness ratio, provides a handle on X-ray absorption (see Sect. 3.2 for further details).

The High Energy X-ray Timing Experiment (HEXTE; Rothschild et al. 1998) is sensitive for X-ray photons between 15 and $250 \mathrm{keV}$ and consisted of two identical clusters, HEXTE-A and -B. These clusters alternated between the on-source position and two background positions. This so-called "rocking" mechanism allowed one to simultaneously measure the X-ray source and background. Due to mechanical failures of this technique late in the mission, cluster A was fixed in the on-source position during all observations of GX 304-1, while cluster B was fixed in one background position $1.5^{\circ}$ off-source. In order to investigate background corrected spectra for GX 304-1, we have extracted the source spectrum from cluster A and estimated its corresponding background from the background spectrum of cluster B using the hextebackest tool as described in Pottschmidt et al. (2006) and R17, Appendix A. The resulting HEXTE-spectra of GX 304-1 have been analysed in the 20-100 keV energy range, no spectral binning was applied, and no systematic uncertainties have been added to the data.

\section{DATA ANALYSIS}

\subsection{Absorption column density}

We have used the Interactive Spectral Interpretation System (ISIS Houck \& Denicola 2000) v1.6.2-30 to perform a combined spectral analysis of the PCA- and HEXTE-spectra for each RXTE observation. We use the results of R17, who describe the RXTE spectra with two different continuum models. The cutoffpl model consists of a power-law in combination with a multiplicative exponential and an additional blackbody (CUTOFFPL + BBODY). The second model highecut is a power-law continuum with an exponential roll-over, which sets in at higher photon energies (POWERLAW $\times$ HIGHECUT). Both models take low energy X-ray absorption into account using the model TBnew ${ }^{1}$ which is and extended version of the model by Wilms et al. (2000) with interstellar element abundances. Here, the corresponding cross-sections were set to those of Verner et al. (1996). The emission lines of iron at $6.40 \mathrm{keV}$ and $7.06 \mathrm{keV}$ were modelled with Gaussians (GAUSS), and the cyclotron line known to be present in GX 304-1 around 50-55 keV (Yamamoto et al. 2011) was described by a multiplicative Gaussian absorption component (GAUABS).

Furthermore, as discussed by R17 several systematic features are detectable in the RXTE spectra. Although the background for

\footnotetext{
1 http://pulsar.sternwarte.uni-erlangen.de/wilms/ research/tbabs/
}

HEXTE-A can be estimated from HEXTE-B, the true background still differs from the estimated one. This results in four additional background lines at 30.17, 39.04, 53.0, and $66.64 \mathrm{keV}$, which we have modelled by additional Gaussian components. In some observations slight residuals in absorption are seen in PCA below $\sim 4.5 \mathrm{keV}$, which we attribute to an insufficient modelling of the Xenon L-edge and have modelled by a negative Gaussian fixed at $3.88 \mathrm{keV}$. A similar systematic feature appears at $10.5 \mathrm{keV}$ for very high source fluxes, which we included in the model as well. Finally, we have introduced a flux calibration constant for HEXTE with respect to PCA and allowed the fit to adapt the background strength for both detectors (CORBACK in ISIS, which is similar to RECOR in XSPEC). A detailed discussion of these systematic features in the RXTE spectra, in particular the HEXTE background lines, is given in R17, Appendix C.

The resulting evolution of the spectral parameters, especially those of the power-law continuum and cyclotron line, for both continuum models (cutoffpl and highecut, see above) is presented in R17. In this work, we focus on the time evolution of the absorption column density, $N_{\mathrm{H}}$, which tracks the number of particles along the line of sight to the neutron star. As already noticed by R17 a large enhancement of the column density is detected during the 2011 May outburst (see Fig. 1b), where $N_{\mathrm{H}}$ is about $\times 3$ higher than what is usually observed in GX 304-1 with RXTE. This enhancement event lasts for around 3 days until $N_{\mathrm{H}}$ suddenly drops to the usual value. A weaker event $(\times 1.5)$ is also visible during the 2010 December outburst. This result is independent of the choice of continuum model (cutoffpl and highecut) and, thus, it is unlikely that the low energy continuum of the neutron star has been modelled improperly during these enhancement events. We will restrict the following discussions to the results of the cutoffpl model.

\subsection{Light curve analysis}

In order to confirm the enhancement in $N_{\mathrm{H}}$ in an independent way of any phenomenological modelling of the X-ray spectra, we have investigated the energy resolved RXTE light curves of GX 304-1. Since material in the line of sight results in absorption of X-ray photons at energies $\lesssim 10 \mathrm{keV}$, the ratio between two light curves in different energy bands, a so-called hardness ratio, then tracks absorption variability without analyzing the full X-ray spectrum. We have computed the ratio of two PCA light curves (1 s time resolution) in the energy bands $2.9-7.7 \mathrm{keV}$ and $7.7-30.0 \mathrm{keV}$. Due to the energy dependence of GX 304-1's pulse profile (see, e.g., Jaisawal et al. 2016), residual pulsations are still visible in the resulting hardness ratio over time, but the pulsed fraction is lower by a factor of 3-5 than compared to the individual light curves.

Figure 1c shows the resulting mean hardness ratio for each observation as determined from the energy resolved light curves. The hardness as defined via the energy bands given above is between 1.0 and 1.8 during most observations. At the same time when the enhancement in the absorption column density is observed during the 2011 May outburst (Fig. 1b), the hardness suddenly increases up to $\sim 2.5$, i.e., by a factor of $\sim 1.4$. Outside of the enhancement event the hardness is again consistent with the data from the other outbursts. This confirms the results of our spectral analysis.

We also found that the standard deviation of the hardness ratio over time during each observation (Figure 1d) shows a very similar increase by a factor of $\sim 1.5$ during the 2011 May outburst. We interpret this variability as evidence for inhomogeneities within the absorbing material in the line of sight, such as a clumpy wind (see, e.g., Hemphill et al. 2014). 


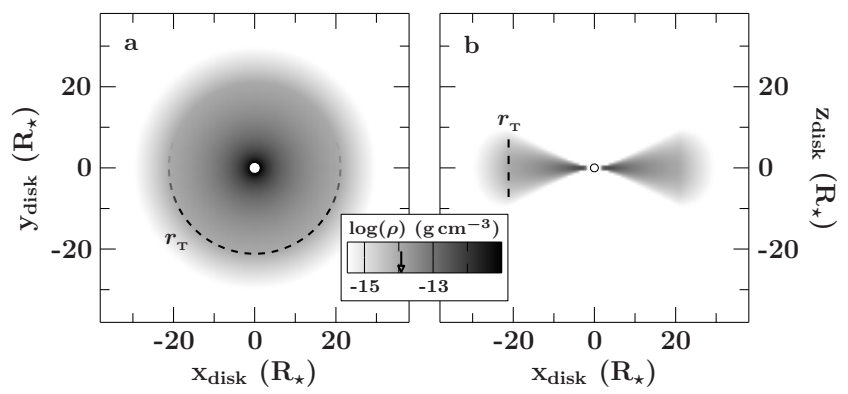

Figure 2. Assumed density profile of the Be disk as calculated after Eqs. 1-4 for a base density of $\rho_{0}=10^{-11} \mathrm{~g} \mathrm{~cm}^{-3}$, a truncation radius $r_{\mathrm{T}} / a=0.49$, and a semi-major axis of $a=601 \mathrm{lt}$-s in Cartesian coordinates $\left(x_{\text {disk }}, y_{\text {disk }}\right.$, $\left.z_{\text {disk }}\right)$. The white circle in the disk's centre is the Be star. The arrow in the density scale marks the density at the truncation radius. a) Density profile within the disk plane $\left(z_{\text {disk }}=0\right)$ showing the radial dependence. b) Height dependence of the density profile, i.e., perpendicular to the disk plane $\left(y_{\text {disk }}=0\right)$.

\section{POSSIBLE OCCULTATION BY THE BE DISK}

In order to investigate the origin of these enhancement events, we have used the orbital parameters by Sugizaki et al. (2015, see Table 1) to convert the date of each RXTE observation into an orbital phase. Figure $1 \mathrm{~b}$ shows the measured absorption column density during all four outbursts over the resulting orbital phases. The two enhancement events nicely align between the orbital phases -0.03 and -0.01 , which is right before the periastron passage of the neutron star. In these events no significant flare was detected on top of the Swift-BAT light curve of GX 304-1 (compare Fig. 1a), which is in agreement with the RXTE light curves (see Fig. 1 of R17). Thus, it is unlikely that the matter, which is responsible for the enhancement in $N_{\mathrm{H}}$, was accreted by the neutron star as this would result in an additional increase in X-ray luminosity.

From GX 304-1's orbital parameters we can estimate the dimension of the cloud of material within the line of sight. Assuming that the material is stationary and neglecting the eccentricity of the neutron star's orbit, we find $2 \pi(a \sin i) \Delta t / P_{\text {orb }} \sim 86$ lt-s as the dimension of the cloud with the duration $\Delta t=3 \mathrm{~d}$ of the enhancement event in 2011 May. Such small scale density fluctuations are not expected in a molecular cloud such as the Coalsack Nebula. Thus, the cloud of material has to be located within the GX 304-1 system. The enhancement event took place right before the periastron passage of the neutron star. The location of the periastron within the binary system is determined by its longitude, $\omega=130^{\circ}$ (see Table 1), which means that this point, as seen from Earth, is behind the companion's tangent plane of the sky. That is, the neutron star is farther away from Earth during the periastron passage than the Be companion. Thus, the X-ray line of sight, which is always fixed on the neutron star, might has passed through or behind the circumstellar material of the companion, such as its equatorial disk.

In this Section, we investigate the possibility of an occultation event of the neutron star by the Be disk. Therefore we introduce a simple 3D-model of a rigid Be disk with a physically motivated density profile and apply this model to the observed $N_{\mathrm{H}}$ evolution.

\subsection{Density profile of the Be disk}

According to the viscous decretion disk scenario (Lee et al. 1991), Be disks are formed by viscous diffusion of gas ejected from the central star. They are Keplerian disks, radially supported by the rotation, and in hydrostatic equilibrium in the vertical direction, supported by the gas pressure. As the model of the Be disk in GX 304-1, we assume for simplicity that the disk is isothermal at $T_{\mathrm{d}}=0.6 T_{\text {eff }}$ (Carciofi \& Bjorkman 2006). We also assume that the disk does not change over the X-ray activity period and its density profile, $\rho(r, h)$, is cylindrically symmetric, i.e., depending on the distance, $r$, to the symmetry axis of the disk and the height, $h$, above the disk plane:

$\rho(r, h)=\rho_{0}\left(\frac{r}{R_{*}}\right)^{-n} \exp \left[-\frac{h^{2}}{2 H(r)^{2}}\right]$,

where $\rho_{0}$ is the base density, i.e., the density at $(r, h)=\left(R_{*}, 0\right), n$ is a constant that characterizes the radial density distribution (see, e.g., Okazaki et al. 2013), $R_{*}$ is the radius of the Be star, and $H(r)$ is the vertical scale-height given by

$H(r)=\frac{c_{\mathrm{S}}}{\Omega_{\mathrm{K}}(r)}=\left(\frac{k T_{\mathrm{d}} R_{*}^{3}}{\mu m_{\mathrm{H}} G M_{*}}\right)^{1 / 2}\left(\frac{r}{R_{*}}\right)^{3 / 2}$,

where $c_{\mathrm{S}}=\left(k T_{\mathrm{d}} / \mu m_{\mathrm{H}}\right)^{1 / 2}$ is the isothermal sound speed, with $\mu$ and $m_{\mathrm{H}}$ being the mean molecular weight and the mass of the hydrogen atom, respectively, and $\Omega_{\mathrm{K}}=\left(G M_{*} / R_{*}^{3}\right)^{1 / 2}$ is the Keplerian rotation velocity with the mass, $M_{*}$, of the Be star. We adopt $R_{*}=6 \mathrm{R}_{\odot}, M_{*}=10 \mathrm{M}_{\odot}$, and $T_{\mathrm{eff}}=22000 \mathrm{~K}$ consistent with a B2Vne star (Parkes et al. 1980) and $\mu=0.62$ for fully ionized plasma with cosmic abundances. We fixed the density profile index $n=2.5$ as expected for a truncated disk as described in the following.

In a binary like GX 304-1, the Be disk is thought to be truncated at a radius smaller than the binary separation at periastron. If the Be disk is coplanar with the binary orbital plane, the truncation occurs at a resonance radius, $r_{\mathrm{T}}$, which mainly depends on the orbital eccentricity and the disk viscosity (Negueruela \& Okazaki 2001; Okazaki \& Negueruela 2001). For instance, for the viscosity parameter $\alpha \sim 0.1$ and the above stellar and disk parameters for GX 304-1, the disk is truncated at the 6:1 resonance radius $\left(r_{\mathrm{T}} / a=0.29\right)$. If the Be disk is highly misaligned as we consider for GX 304-1, however, it has a significantly larger radius than the coplanar disks, because of the weaker resonant torques, and is likely to fill the Roche lobe of the Be star (Lubow et al. 2015; Miranda \& Lai 2015). Given that there are large uncertainties in the viscosity parameter (according to Clark et al. 2001 and Wisniewski et al. 2010, $\alpha \sim 0.1$, while Carciofi et al. 2012 assume $\alpha \sim 1$ ) and the misalignment angle, we examine the disk obscuration effect for two extreme disk cases, one truncated at the $6: 1$ resonance radius $\left(r_{\mathrm{T}} / a=0.29\right)$ and the other that fills the Roche lobe radius averaged over the binary orbit $\left(r_{\mathrm{T}} / a=0.49\right)$. In the calculation of the latter radius, we applied the approximated formula by Eggleton (1983) to the averaged binary separation, $d=a\left(1-e^{2}\right)^{1 / 2}$, as

$r_{\mathrm{T}}=\frac{0.49 q^{2 / 3} d}{0.6 q^{2 / 3}+\ln \left(1+q^{1 / 3}\right)}$,

where $q=M_{*} / M_{\mathrm{X}}$ is the binary mass ratio assuming the canonical neutron star mass of $M_{\mathrm{X}}=1.4 \mathrm{M}_{\odot}$. For radii larger than the truncation radius, $r_{\mathrm{T}}$, we assume that the density distribution of the $\mathrm{Be}$ disk is

$\rho\left(r>r_{\mathrm{T}}, h\right)=\rho_{\mathrm{T}}(h)\left(\frac{r}{r_{\mathrm{T}}}\right)^{-m}$

with the density at the truncation radius $\rho_{T}(h)=\rho\left(r_{\mathrm{T}}, h\right)$ as calculated after Eq. 1. The constant $m>n$ leads to a faster decrease of the density profile than for radii $r<r_{\mathrm{T}}$ and we assume $m=10$, which is consistent with numerical results obtained by Okazaki et al. (2002). 
Figure 2 shows the radial (a) and height (b) dependence of the density profile as defined above in the case of $r_{\mathrm{T}} / a=0.49$ with $a=601 \mathrm{lt}-\mathrm{s}$ and $\rho_{0}=10^{-11} \mathrm{~g} \mathrm{~cm}^{-3}$.

\subsection{Density along the X-ray line of sight}

In order to calculate the absorption column density, $N_{\mathrm{H}}$, we need to transform the line of sight to the neutron star, given in the binary's reference frame, into the Be-disk's reference frame, i.e., into cylindrical coordinates. Figure 3 shows a sketch of the following definitions. We define the origin of the binary's reference frame to be fixed at the position of the Be-type companion star. The xy-plane is equivalent to the tangent plane of the sky, i.e., the $z$-axis is parallel to the line of sight to the companion and pointing away from Earth. The position of the neutron star on its orbit is found by solving Kepler's equation,

$E-e \sin E=M$,

for the eccentric anomaly, $E$. Here, $e$ is the eccentricity of the orbit and

$M=2 \pi\left(\frac{t-\tau}{P_{\text {orb }}}\right)$

is the mean anomaly with the time of the observation, $t$, the time of periastron passage, $\tau$, and the orbital period, $P_{\text {orb }}$ (see Table 1 for the orbital parameters of GX 304-1). The position vector to the neutron star, $\boldsymbol{r}_{\mathrm{ns}}=\left(x_{\mathrm{ns}}, y_{\mathrm{ns}}, z_{\mathrm{ns}}\right)^{\mathrm{T}}$, is then given by

$$
\left(\begin{array}{l}
x_{\mathrm{ns}} \\
y_{\mathrm{ns}} \\
z_{\mathrm{ns}}
\end{array}\right)=\mathbf{R}\left(\boldsymbol{e}_{\mathrm{y}}, 90^{\circ}-i\right)\left(\begin{array}{c}
0 \\
a \cos \omega(\cos E-e)-b \sin E \sin \omega \\
a \sin \omega(\cos E-e)+b \sin E \cos \omega
\end{array}\right)
$$

with the rotation matrix, $\mathbf{R}$, around the unit vector along the $y$-axis, $\boldsymbol{e}_{\mathrm{y}}$, and the angle $90^{\circ}-i$ with the inclination, $i$, of the orbital plane with respect to the tangent plane of the sky. The semi-major axis, $a$, is found using the measured value of $a \sin i, b=\sqrt{a^{2}\left(1-e^{2}\right)}$ is the semi-minor axis, and $\omega$ is the argument of periastron.

In order to transform any vector in the binary's reference frame into the reference frame of the Be disk with cylindrical coordinates $(r, h)$, we first calculate the normal vector, $\boldsymbol{n}_{\text {disk }}$, of the disk by taking the inclination of the orbit, $i$, the misalignment angle of the disk, $\delta$, and the position angle of the disk, $\theta$, into account,

$\boldsymbol{n}_{\text {disk }}=\mathbf{R}\left(\boldsymbol{n}_{\mathrm{orb}}, \theta\right) \mathbf{R}\left(\boldsymbol{e}_{\mathrm{y}}, 90^{\circ}-i+\delta\right) \boldsymbol{e}_{\mathrm{x}}$.

Here, $\boldsymbol{e}_{\mathrm{X}}$, is the unit vector along the $x$-axis (of the binary's reference frame), and $\boldsymbol{n}_{\text {orb }}$ is the normal vector of the orbital plane,

$\boldsymbol{n}_{\text {orb }}=\mathbf{R}\left(\boldsymbol{e}_{\mathrm{y}}, 90^{\circ}-i\right) \boldsymbol{e}_{\mathrm{x}}$.

Due to the fact that no enhancement event was detected in the earlier outbursts of 2010, the Be disk was not in the X-ray line of sight, while a strong occultation event occurred in 2011 May. A likely explanation is that the Be disk in GX 304-1 is precessing. Since we expect the Be disk to precess in the retrograde direction compared to the orbital movement of the neutron star (Papaloizou \& Lin 1994; Papaloizou \& Terquem 1995), we calculate the position angle of the disk after

$\theta=\omega_{\mathrm{disk}}-\dot{\omega}_{\mathrm{disk}}\left(t-t_{0}\right)$,

with the initial position angle $\omega_{\text {disk }}$ at the time $t_{0}$ and the precession frequency, $\dot{\omega}_{\text {disk }}$. Note that in the context of the binary's reference frame defined above, the position angle, $\theta$, is measured between the line of sight, $\boldsymbol{e}_{\mathrm{Z}}$, and the highest point of the disk, both projected onto the orbital plane (see Fig. 3). Finally, the transformation, $T$, of any
Table 2. Parameters of the Be-disk occultation model as defined in Eqs. 512. See the text for a detailed description and for the assumptions of fixing certain parameters.

\begin{tabular}{lll}
\hline Be-star radius & $R_{*}=6 \mathrm{R}_{\odot}$ & (fixed) \\
Be-star mass & $M_{*}=10 \mathrm{M}_{\odot}$ & (fixed) \\
Be-disk temperature & $T_{\text {eff }}=22000 \mathrm{~K}$ & (fixed) \\
Be-disk truncation radius (see text) & $r_{\mathrm{T}}$ & (fixed) \\
Be-disk base density & $\rho_{0}$ & \\
Density profile index & $n=2.5$ & (fixed) \\
Density profile index (truncated) & $m=10$ & (fixed) \\
Orbital parameters (see Table 1) & $P_{\text {orb }}, a \sin i, e, \tau, \omega$ & (fixed) \\
Orbit inclination & $i$ & \\
Be-disk inclination & $\delta$ & \\
Be-disk position angle at $t_{0}$ & $\omega_{\text {disk }}$ & \\
Be-disk precession frequency & $\dot{\omega}_{\text {disk }}$ & \\
Precession reference time & $t_{0}=$ MJD 55690 & (fixed) \\
Foreground absorption & $N_{\mathrm{H}, \text { frgrd }}$ & \\
\hline
\end{tabular}

vector, $\boldsymbol{r}$, given in the binary's reference frame into the cylindrical coordinates of the reference frame of the Be disk is

$T: \quad \boldsymbol{r} \rightarrow(r, h)=\left(\left|\boldsymbol{r}-\left(\boldsymbol{n}_{\mathrm{disk}} \cdot \boldsymbol{r}\right) \boldsymbol{n}_{\mathrm{disk}}\right|, \boldsymbol{n}_{\mathrm{disk}} \cdot \boldsymbol{r}\right)$.

The absorption column density is finally found by computing the integral of the Be-disk density, $\rho$ (Eqs. 1-4), along the line of sight, i.e., along the $z$-axis up to the neutron star's position:

$N_{\mathrm{H}}=N_{\mathrm{H}, \text { frgrd }}+\frac{1}{m_{\mathrm{H}}} \int_{-\infty}^{z_{\mathrm{ns}}} \rho\left(T\left(\left(x_{\mathrm{ns}}, y_{\mathrm{ns}}, z^{\prime}\right)^{\mathrm{T}}\right)\right) \mathrm{d} z^{\prime}$

Here, $N_{\mathrm{H} \text {,frgrd }}$ accounts for constant interstellar foreground absorption. Table 2 summarizes the fixed and free parameters of the full model.

\subsection{Modelling the observed absorption column densities}

Initial attempts to apply the model defined in Eq. 12 to the observed $N_{\mathrm{H}}$ evolution over time shown in Fig. $1 \mathrm{~b}$ revealed several issues. First, finding the best-fit using a commonly used $\chi^{2}$-minimization is complicated by several local minima within the $\chi^{2}$-landscape, where the actual observed enhancement event in 2011 May is not modelled at all. Furthermore, $\chi_{\text {red }}^{2}$ is much larger than unity even during outbursts where no occultation event is detected due to a significant scattering of the observed $N_{\mathrm{H}}$ values (see discussion below). Finally, from the calculation of the disk's normal vector, $\boldsymbol{n}_{\text {disk }}$ (Eq. 8), we expect a parameter degeneracy between the orbit inclination, $i$, and the Be-disk inclination, $\delta$.

In order to solve these issues, we applied a Bayesian analysis of the model to the $N_{\mathrm{H}}$ evolution in form of a Markov chain Monte Carlo (MCMC) sampling approach after Goodman \& Weare (2010). We used the emcee algorithm as implemented by ForemanMackey et al. (2013) and ported into ISIS by M.A. Nowak, which is distributed via the ISISscripts ${ }^{2}$. The advantages of this approach are that parameter degeneracies are automatically error propagated and the algorithm always provides the most probable answer even in cases of a bad goodness of the fit. The result of an emcee run is the probability distribution for each free parameter of the model used. The most probable parameters, i.e., the best-fit parameters, correspond to the maxima found in the probability distributions. These are sampled by so-called walkers, which move within the parameter space and are distributed uniformly at the beginning.

${ }^{2}$ http://www.sternwarte.uni-erlangen.de/isis 
a)

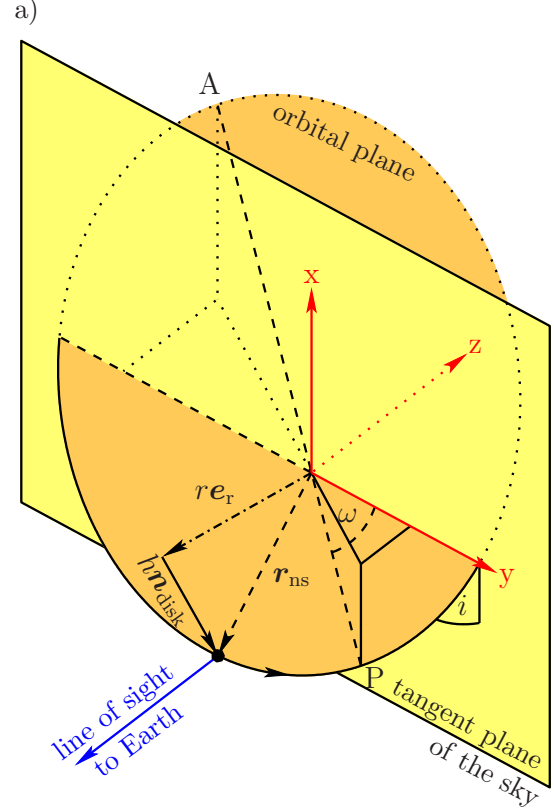

b)

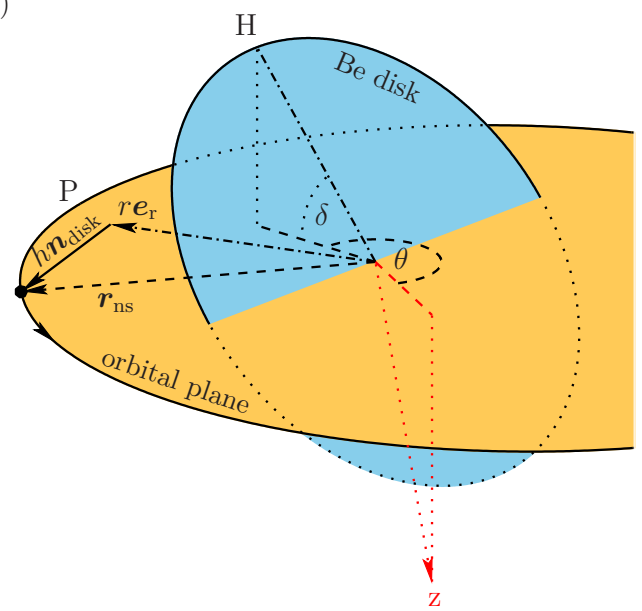

Figure 3. Definition of the reference frames as described in the text. a) The tangent plane of the sky (yellow) defines the cartesian reference frame ( $x, y, z$; red arrows) with the $z$-axis and the line of sight (blue arrow) perpendicular to this plane. Dashed lines are within the orbital plane (orange). $\mathrm{P}$ and A denote the position of the periastron and apastron on the neutron star orbit, respectively. b) The Be disk (blue) is inclined with respect to the orbital plane (orange). The position vector of the neutron star $\left(\boldsymbol{r}_{\mathrm{ns}}\right)$ is decomposed using the unit vectors of the Be-disk plane $\left(\boldsymbol{e}_{\mathrm{r}}\right.$ and $\left.\boldsymbol{n}_{\text {disk }}\right)$. Dash-dotted lines are within the Be-disk plane. $\mathrm{H}$ marks the highest point of the disk above the orbital plane. Note that for clarity we show different orbital phases in plots a) and b).

For each possible truncation radius $\left(r_{\mathrm{T}} / a=0.49\right.$ or 0.29 , see Sect. 4.1), we have performed an emcee run with 1000 walkers per free parameter and 10000 iteration steps. The resulting parameter chains were investigated on convergence, i.e, the acceptance rate is stable around 0.25 after $\sim 500$ iteration steps, which is in the range of $0.2-0.5$ as expected in convergence (Foreman-Mackey et al. 2013). Thus, we ignored the first 500 iteration steps in the following analysis of the parameter distributions.

Almost all probability distributions, which are found by sorting the corresponding parameter chain into a histogram, show two or three distinct maxima, i.e., multiple possible solutions. These solutions are best seen in the 2D-probability distribution (i.e., similar to a $\chi^{2}$ contour map) between the orbit inclination, $i$, and the Be-disk inclination, $\delta$. Figure 4 shows this distribution for a Be-disk truncation radius of $r_{\mathrm{T}} / a=0.49$. The peak with the highest probability (solution \#1) corresponds to a high disk misalignment angle, $\delta$, and a moderate orbit inclination, $i$, while its the other way around for the second highest peak (solution \#2). Furthermore, the degeneracy between these parameters as expected from Eq. 8 is visible as diagonal valleys in the figure. In the case of $r_{\mathrm{T}} / a=0.29$ even a third solution appears (solution \#3).

In order to reduce the influence of these solutions on the 1Dprobability distributions, which are used to derive the parameter values and uncertainties, we have split the parameter chains into areas around each solution. Fig. 4 shows these areas for the case of $r_{\mathrm{T}} / a=0.49$. In this way, the number of peaks in the probability distribution of each parameter reduces to one, i.e., the solution is unique and we are able to provide the final parameter values for all possible solutions and the two extreme cases for the truncation radius. The most probable parameter value is found by determining the peak's position using a polynomial fit around the maximum. The lower and upper confidence levels are determined such that $68 \%$ of the peak's total area is within this confidence interval. Thereby, a

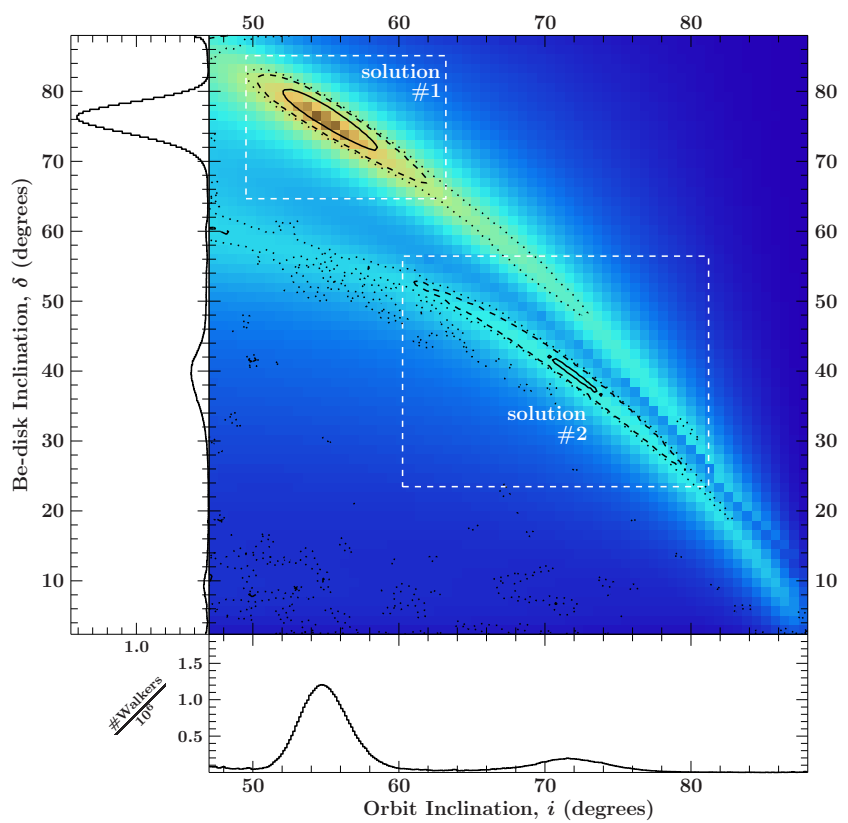

Figure 4. 2D-probability contours (black) between the orbit inclination, $i$, and the Be-disk inclination, $\delta$, with respect to the orbital plane for the case $r_{\mathrm{T}} / a=0.49$. The line style indicates the $68 \%$ (solid), $90 \%$ (dashed), and $99 \%$ (dotted) confidence level. In order to check the emcee result, the underlying colour map shows the corresponding $\chi^{2}$ at each point, calculated independently of the emcee run. The vertical and horizontal histograms correspond to the $1 \mathrm{D}$-probability distributions of both parameters, i.e., summing up the columns or rows of the map, respectively. The white, dashed boxes mark the regions which have been used to split the parameter chains into the two distinct solutions (\#1 and \#2). 
Table 3. Most probable parameters for both extreme cases of Be-disk truncation and for all solutions discovered in the parameter space. See Table 2 for a brief description of the model parameters.

\begin{tabular}{lllllll}
\hline $\begin{array}{l}r_{\mathrm{T}} / a \text { [solution no.] } \\
\text { Parameter }\end{array}$ & (unit) & $0.49[\# 1]$ & $0.49[\# 2]$ & $0.29[\# 1]$ & $0.29[\# 2]$ & $0.29[\# 3]$ \\
\hline$i$ & (degree) & $54.7_{-1.5}^{+1.9}$ & $71.7_{-2.3}^{+2.6}$ & $67.1_{-0.8}^{+0.8}$ & $82.5_{-1.4}^{+1.1}$ & $54.5_{-1.7}^{+1.7}$ \\
$\delta$ & (degree) & $76.3_{-2.5}^{+2.0}$ & $40_{-5}^{+4}$ & $62.7_{-1.5}^{+1.3}$ & $17.8_{-2.8}^{+3.1}$ & $81.9_{-2.3}^{+2.0}$ \\
$\omega_{\text {disk }}$ & (degree) & $121.5_{-0.8}^{+0.8}$ & $89.4_{-1.5}^{+2.0}$ & $138.0_{-1.8}^{+1.9}$ & $74.5_{-2.4}^{+2.4}$ & $131.9_{-2.6}^{+2.5}$ \\
$\dot{\omega}_{\text {disk }}$ & $\left(\right.$ degree $\left.\mathrm{yr}^{-1}\right)$ & $190_{-5}^{+4}$ & $250_{-6}^{+5}$ & $135_{-13}^{+34}$ & $271_{-10}^{+8}$ & $176_{-5}^{+5}$ \\
$\log \left(\rho_{0} / \mathrm{g} \mathrm{cm}^{-3}\right)$ & & $-10.29_{-0.05}^{+0.04}$ & $-10.62_{-0.07}^{+0.06}$ & $-10.22_{-0.06}^{+0.06}$ & $-10.81_{-0.05}^{+0.06}$ & $-9.37_{-0.13}^{+0.16}$ \\
$N_{\mathrm{H}, \text { frgrd }}$ & $\left(10^{22} \mathrm{~cm}^{-2}\right)$ & $3.26_{-0.13}^{+0.13}$ & $3.22_{-0.15}^{+0.18}$ & $3.79_{-0.11}^{+0.11}$ & $3.64_{-0.13}^{+0.29}$ & $3.75_{-0.12}^{+0.14}$ \\
$\chi^{2} /$ d.o.f. & & $252.03 / 63$ & $303.11 / 63$ & $313.08 / 63$ & $290.51 / 63$ & $307.33 / 63$ \\
\hline
\end{tabular}

linear continuum has been subtracted from the probability distribution in order to investigate the area of the peak only. Table 3 lists the resulting most probable parameters and uncertainties for each investigated truncation radius and solution found in the parameter space. For the case of $r_{\mathrm{T}} / a=0.49$, we compare in Fig. 5 the modelled evolution of $N_{\mathrm{H}}$ for both solutions with the data. We also show the resulting geometries of the binary and Be disk projected onto the tangent plane of the sky, i.e., as seen from Earth.

\section{DISCUSSION \& CONCLUSIONS}

In this paper we have presented a study of the $N_{\mathrm{H}}$ behaviour of GX 304-1 during four outbursts in terms of an occultation by a precessing Be-star disk. The most probable parameters listed in Table 3 correspond to the different solutions discovered in the parameter space.

Formally, there are five solutions across the two truncation radii - two for $r_{\mathrm{T}} / a=0.49$ and three for $r_{\mathrm{T}} / a=0.29$. However, these really reflect only three basic physical scenarios, which we refer to as solutions \#1, \#2, and \#3. Solution \#1 and \#2 are found for both $r_{\mathrm{T}} / a=0.49$ and $r_{\mathrm{T}} / a=0.29$, while Solution \#3 only appears in the $r_{\mathrm{T}} / a=0.29$ case. Solution \#1 corresponds to a system with moderate orbital inclination and a highly-misaligned, high-density Be-disk which precesses relatively slowly. Solution \#2 has a higher inclination, but a less-misaligned, lower-density Be-disk precessing more quickly. Finally, solution \#3 has a very highly misaligned, very high-density Be-disk in a system with a precession frequency and inclination comparable to solution \#1.

We have investigated additional truncation radii and found that the parameters of solutions \#1 and \#2 change nonlinearly with truncation radius. As the truncation radius is decreased, solution \#3 migrates into the parameter space, starting at very high disk misalignment angles (the upper left corner in Fig. 4). Which solution is the most realistic cannot be decided easily, as discussed in the following.

The best nominal solution in terms of $\chi^{2}$ is a large Be disk with a high misalignment angle $\left(r_{\mathrm{T}} / a=0.49\right.$, \#1, first column in Table 3). From the statistical point of view this solution is, however, still not acceptable $\left(\chi_{\text {red }}^{2}=4.27\right)$. The unacceptable high $\chi^{2}$ values may have several reasons: first, our simple model assumes a rigid and cylindrically symmetric Be disk. In particular, we ignore any structures within the Be disk, such as warping and spiral density waves, which are known to be present due to the tidal interaction with the neutron star (see, e.g., Okazaki et al. 2002 and Martin et al. 2011). Such a behaviour might explain the observed scattering of the
$N_{\mathrm{H}}$ values, especially during the 2010 December outburst. Here, our most probable model suggests that the line of sight crosses the outermost parts of the Be disk near the truncation radius (Fig. 5), where the tidal effects are expected to be most prominent as discussed for the BeXRB 4U 0115+634 by Negueruela \& Okazaki (2001) and Reig et al. (2007) and for A0535+262 by Moritani et al. (2013). Secondly, the $N_{\mathrm{H}}$ measurements itself might be influenced by systematic effects, such as the Xe L-edge, which R17 had to introduce in order to model calibration uncertainties in RXTE-PCA. Further evidence for these systematics is that the emcee runs prefer a large interstellar foreground absorption of $N_{\mathrm{H} \text {,frgrd }} \sim 3.6 \times 10^{22} \mathrm{~cm}^{-2}$. Although GX 304-1 is located in direction of the Coalsack nebula, Suzaku observations during its 2010 August and 2012 January outbursts revealed $N_{\mathrm{H}} \sim 1 \times 10^{22} \mathrm{~cm}^{-2}$ (Jaisawal et al. 2016), which is consistent with the foreground absorption found in $21 \mathrm{~cm}$ surveys (Kalberla et al. 2005; Dickey \& Lockman 1990). Assuming a systematic uncertainty of $2 \times 10^{22} \mathrm{~cm}^{-2}$ to be consistent to these alternative foreground absorption measurements indeed results in a $\chi_{\text {red }}^{2}$ around unity. This does not, however, affect the significance of the occultation event in 2011 May.

We have found that the base density of the Be disk, $\rho_{0}$, which is mainly determined by the measured $N_{\mathrm{H}}$ values during the occultation event in 2011 May, is on the order of $10^{-11}-10^{-10} \mathrm{~g} \mathrm{~cm}^{-3}$. This result is in very good agreement with the commonly accepted value of $10^{-11} \mathrm{~g} \mathrm{~cm}^{-3}$ (e.g., Okazaki et al. 2013) for disks of isolated Be stars. Although the Be disks in binaries are expected to be approximately twice as dense as compared to isolated stars (Zamanov et al. 2001), the solutions with densities significantly above $10^{-10} \mathrm{~g} \mathrm{~cm}^{-3}$ would be unlikely, such as solution \#3 with $r_{\mathrm{T}} / a=0.29$.

The precession period, $\dot{\omega}_{\text {disk }}$, of solution \#2 is about $30 \%-$ $100 \%$ faster than that of solution \#1. This difference decreases with increasing truncation radius. According to Larwood (1998), the precession period, $P_{\text {disk }}$, due to tidal forces on a misaligned disk is

$P_{\text {disk }}=\frac{7}{3}\left(\frac{1+q}{q^{2}}\right)^{1 / 2}\left(\frac{a}{r_{\mathrm{T}}}\right)^{3 / 2} \frac{P_{\text {orb }}}{\cos \delta}$

with $q=M_{\mathrm{X}} / M_{*}$. Using the resulting disk misalignment angle, $\delta$, of solution \#1 for the truncation radius $r_{\mathrm{T}} / a=0.49$ we find $P_{\text {disk }} \sim 80 \mathrm{yr}$, which is $\times 42$ longer than the emcee result of $360^{\circ} / \dot{\omega}_{\text {disk }} \sim 1.9$ yr. Using other emcee solutions does not weaken this disagreement significantly. Since the material forming the Be disk originates from the central Be star, the disk's precession might be induced by an intrinsically precessing Be star. This precession is the result of tidal forces on an oblate star such as a rapidly rotating Be star. Using the theoretical investigations by Kopal (1959) and Alexander (1976), we have estimated the precession period of the 

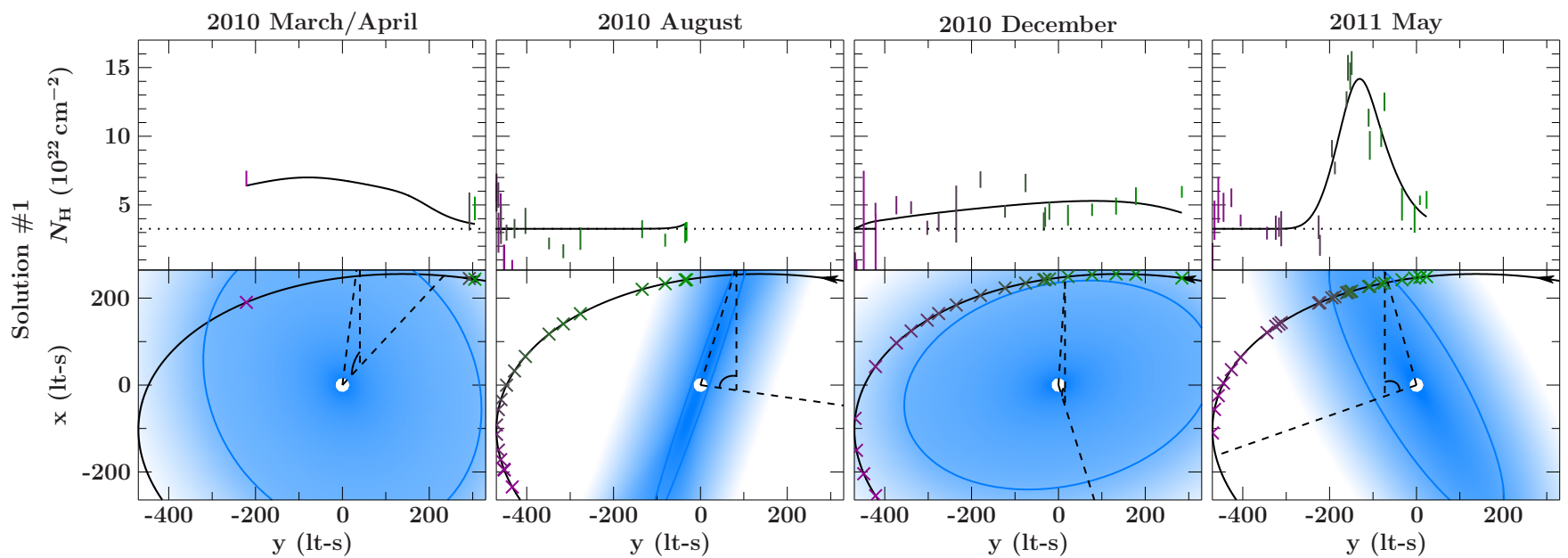

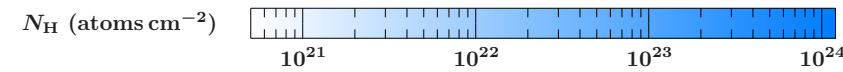
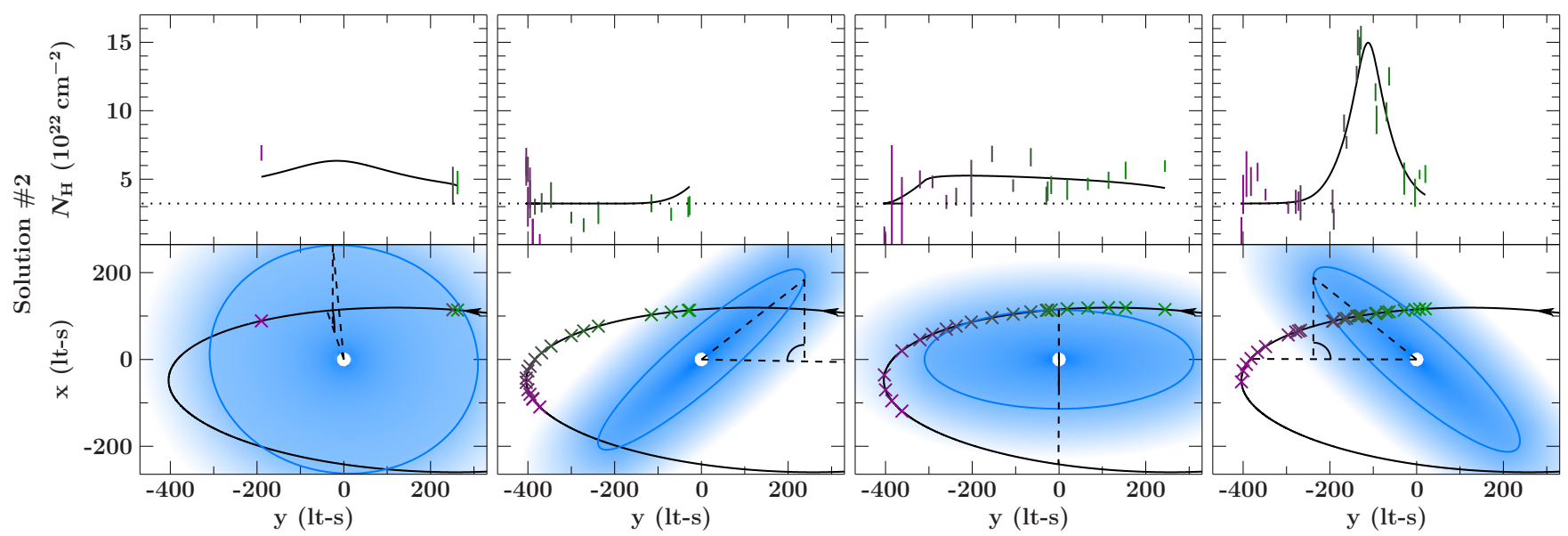

Figure 5. Absorption column and projected geometry of the Be disk for the case of $r_{\mathrm{T}} / a=0.49$. The upper row shows the results for solution \#1, the lower row presents the results for solution \#2. Results for $r_{\mathrm{T}} / a=0.29$ are not shown as they are very similar. The top panels in each subfigure show measured (green/purple) and modelled (black) absorption column densities, $N_{\mathrm{H}}$, for all four outbursts of GX 304-1 (each column represents one outburst). Colours of data points indicate the time of the observation during a certain outburst (from green to purple). The dotted line is the most probable interstellar foreground absorption, $N_{\mathrm{H} \text {, frgrnd }}$ (see Table 3). The bottom panels in each subfigure illustrate the modelled Be-disk geometry and neutron star position as seen from Earth, i.e., the components of the binary are projected onto the tangent plane of the sky. The white circle in the centre of the reference frame is the Be star, the crosses mark the positions of the neutron star along its orbit (black line) during all observations. The blueish region is proportional to the $N_{\mathrm{H}}$ along the line sight through the Be disk, and the blue circle marks the truncation radius, $r_{\mathrm{T}} / a=0.49$, of the Be disk within the disk plane. The highest point on the disk's rim above the orbital plane is connected with the central Be star by one dashed line and to the orbital plane by another line perpendicular to the latter. The dashed line from the Be star to the neutron star's orbit marks the orbital phase of the highest point of the disk, i.e., the position angle of the disk, $\omega_{\text {disk }}$.

Be star in GX 304-1 to be on the order of $5000 \mathrm{yr}$ (see appendix A for the detailed calculation). This rules out Be star precession as the origin of the fast Be disk precession we have derived. However, in case of eccentric orbits, as usually present in BeXRBs, the eccentric Kozai-Lidov (KL) mechanism has to be taken into account for the torque on misaligned disks as calculated after Eq. 13. Following the formulation by Naoz et al. (2013) and Naoz (2016), we have numerically investigated the evolution of Be-disk particle orbits with initial radii between $r_{\mathrm{T}} / a=0.49$ and $r_{\mathrm{T}} / a=0.29$ and similar misalignment angles, $\delta$, as listed in Table 3 . As a result from the KL mechanism, the Be-disk particles precess much faster around the binary's orbital momentum vector than compared to the circular approximation of Eq. 13. For a Be-disk radius of $r_{\mathrm{T}} / a=0.29$ the precessing time-scale is $\sim 10 \mathrm{yr}$ and decreases to $4-5 \mathrm{yr}$ for $r_{\mathrm{T}} / a=0.49$ for all misalignment angles, which is in agreement with our best- fit within a factor of 2-3. A very similar time-scale was found in A $0535+262$ by Moritani et al. (2013), who performed an optical spectroscopic monitoring of its Be-type companion star. From the measured radial velocity curve of an enhanced component in the $\mathrm{H} \alpha$ line profile, they have derived a period of $\sim 674 \mathrm{~d}$, which they interpret as the precession period of a warped Be-disk.

The misalignment angles of the Be disk found by our analysis are in the range of $15^{\circ}<\delta<85^{\circ}$. The most probable solution $\left(r_{\mathrm{T}} / a=0.49, \# 1\right)$ points, however, to a large misalignment angle of around $80^{\circ}$. As discussed by Brandt \& Podsiadlowski (1995) a high-velocity supernova kick of the neutron star's progenitor might result in a large misalignment angle. In fact, they suggested that the Be disk in BeXRBs are typically not aligned with the orbital plane. An inclined Be disk in GX 304-1 with respect to the orbital plane was also proposed by Postnov et al. (2015) as one possible explana- 


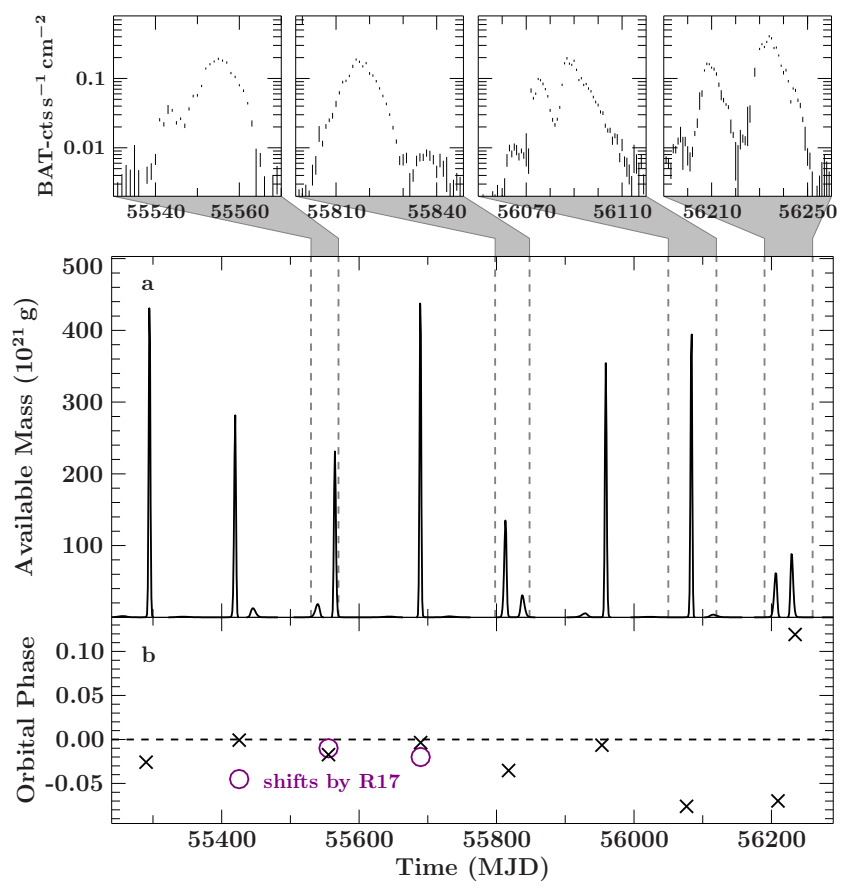

Figure 6. a) The available Be-disk mass within the Roche radius of the neutron star as a function of time for $r_{\mathrm{T}} / a=0.49$ and solution \#1. The plots on top show the observed Swift-BAT light curve of GX 304-1 around some peaks in the predicted available mass. b) The orbital phases (black crosses) for the maxima in a) around the observed outbursts. R17 introduced shifts in orbital phase (purple circles) in order to match the falling edges of the outburst light curves.

tion for the occurrence of the two observed double-peaked outbursts of the source in 2010 June and 2010 October. We have investigated this idea further and calculated the available Be-disk mass within the Roche radius of the neutron star as calculated after Eq. 3 for the mass ratio $q=M_{\mathrm{X}} / M_{*}$ and $d=a\left(1-e^{2}\right) /(1+e \cos (f))$ with the true anomaly, $f$, of the neutron star's position. As shown in Fig. 6 a the resulting mass within the Roche radius around the observed double-peaked outburst of GX 304-1 at MJD 56220 indeed shows two distinct maxima. During this outburst, the orbital phase of the highest point of the disk is near zero, i.e., at the periastron passage of the neutron star. Thus, the neutron star orbit crosses the disk plane twice. This strengthens the idea that double-peaked outbursts are due to a misaligned Be disk, which moves into the orbit of the neutron star as proposed by Postnov et al. (2015) for GX 304-1 and by Okazaki et al. (2013) in general. Furthermore, the model predicts weak leading and trailing flares to the periastron outbursts, which are indeed observed at MJD 55540 and MJD 55830 in the Swift-BAT light curve (shown on top of Fig. 6a). Our model fails to explain the observed double-peaked outburst around MJD 56080 and the available mass during each outburst does not scale with the observed count rate of GX 304-1 as measured with Swift-BAT. The available mass can be modified, however, due to a warped disk, density fluctuations, or tidal streams. The latter results from overflowing gas of the Be disk close to periastron, which gets unbound as soon as the Roche radius of the companion star decreases below the disk's truncation radius, which is the case for $r_{\mathrm{T}} / a \gtrsim 0.30$ in GX 304-1. Finally, deriving the mass accretion rate and, thus, the luminosity from the available mass requires further geometrical and hydrodynamical investigations, which is beyond the scope of this paper.

Figure $6 \mathrm{~b}$ shows the orbital phases at which the available mass within the neutron star's Roche lobe was at a maximum (including both maxima for the double-peaked outburst around MJD 56220). The orbital phases scatter around the periastron passage between -0.08 and +0.12 . For the 2010 August, 2010 December, and 2011 May outbursts of GX 304-1, R17 noticed orbital phase shifts between the falling edges of these outbursts. Interestingly, our derived orbital phases match those of R17 to some extent (Fig. 6b, purple circles).

In summary, a precessing and inclined Be disk explains the

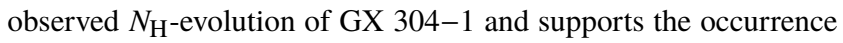
of double-peaked outbursts and phase shifts of the outburst light curves. Our model favours a large Be disk truncated close to the Roche radius for the averaged binary separation. The fast disk precession found can be explained by the eccentric Kozai-Lidov mechanism. However, some issues still remain such as the unacceptable goodness of the fit, the high number of possible solutions, and mismatches between some predicted and observed double-peaked outbursts. We stress that until these issues are solved any numbers derived from our analysis should be taken with care and understood in a qualitative way. Increasing the data sample of observed column densities towards GX 304-1 in combination with optical observation of its companion, in particular $\mathrm{H} \alpha$ line profiles, would help solving these issues in the future.

\section{ACKNOWLEDGEMENTS}

MK acknowledges support by the Bundesministerium für Wirtschaft und Technologie under Deutsches Zentrum für Luft- und Raumfahrt grants 50OR1113 and 50OR1207 and by the European Space Agency under contract number C4000115860/15/NL/IB. SMN acknowledges support by research project ESP2016-76683-C3-1R. JMT acknowledges research grant ESP2014-53672-C3-3P. We thank the Deutscher Akademischer Austauschdienst (DAAD) for funding JC through the Research Internships in Science and Engineering (RISE). Parts of this work can be found in the Ph.D. thesis of Müller (2013). All figures within this paper were produced using the SLXfig module, which was developed by John E. Davis. We thank the RXTE-team for accepting and performing our observations of GX 304-1. Finally, we acknowledge the comments by the referee, which helped improving the content of our paper.

\section{REFERENCES}

Alexander M. E., 1976, Ap\&SS, 45, 105

Brandt N., Podsiadlowski P., 1995, MNRAS, 274, 461

Carciofi A. C., Bjorkman J. E., 2006, ApJ, 639, 1081

Carciofi A. C., Bjorkman J. E., Otero S. A., Okazaki A. T., Štefl S., Rivinius T., Baade D., Haubois X., 2012, ApJ, 744, L15

Clark J. S., Tarasov A. E., Okazaki A. T., Roche P., Lyuty V. M., 2001, A\&A, 380, 615

Dickey J. M., Lockman F. J., 1990, ARA\&A, 28, 215

Eggleton P. P., 1983, ApJ, 268, 368

Finger M. H., et al., 2009, ArXiv Astrophysics e-prints

Foreman-Mackey D., Hogg D. W., Lang D., Goodman J., 2013, PASP, 125, 306

Goodman J., Weare J., 2010, Commun. Appl. Math. Comput. Sci., 5, 65

Hanuschik R. W., 1996, A\&A, 308, 170

Hemphill P. B., Rothschild R. E., Markowitz A., Fürst F., Pottschmidt K., Wilms J., 2014, ApJ, 792, 14 
Houck J. C., Denicola L. A., 2000, in Manset N., Veillet C., Crabtree D., eds, Astron. Soc. of the Pacific Conf. Series Vol. 216, Astronomical Data Analysis Software and Systems IX. p. 591

Jahoda K., Markwardt C. B., Radeva Y., Rots A. H., Stark M. J., Swank J. H., Strohmayer T. E., Zhang W., 2006, ApJS, 163, 401

Jaisawal G. K., Naik S., Epili P., 2016, MNRAS, 457, 2749

Kalberla P. M. W., Burton W. B., Hartmann D., Arnal E. M., Bajaja E., Morras R., Pöppel W. G. L., 2005, A\&A, 440, 775

Klochkov D., et al., 2012, A\&A, 542, L28

Kopal Z., 1959, Close binary systems. The International Astrophysics Series, John Wiley \& Sons Inc., New York

Larwood J., 1998, MNRAS, 299, L32

Lee U., Osaki Y., Saio H., 1991, MNRAS, 250, 432

Lewin W. H. G., Clark G. W., Smith W. B., 1968, Nature, 219, 1235

Liu Q. Z., van Paradijs J., van den Heuvel E. P. J., 2006, A\&A, 455, 1165

Lubow S. H., Martin R. G., Nixon C., 2015, ApJ, 800, 96

Manousakis A., et al., 2008, INTEGRAL hard X-ray detection of HMXB GX 304-1 and H 1417-624, ATel 1613

Martin R. G., Pringle J. E., Tout C. A., Lubow S. H., 2011, MNRAS, 416, 2827

Mason K. O., Murdin P. G., Parkes G. E., Visvanathan N., 1978, MNRAS, $184,45 \mathrm{P}$

McClintock J. E., Nugent J. J., Li F. K., Rappaport S. A., 1977, ApJ, 216, L15

Miranda R., Lai D., 2015, MNRAS, 452, 2396

Moritani Y., et al., 2013, PASJ, 65, 83

Müller S., 2013, PhD thesis, Friedrich-Alexander-Universität ErlangenNürnberg

Naoz S., 2016, ARA\&A, 54, 441

Naoz S., Farr W. M., Lithwick Y., Rasio F. A., Teyssandier J., 2013, MNRAS, 431,2155

Negueruela I., Okazaki A. T., 2001, A\&A, 369, 108

Nyman L.-A., 2008, in Reipurth B., ed., , Handbook of Star Forming Regions, Volume II. Astronomical Society of the Pacific Monograph Publications, p. 222

Okazaki A. T., Negueruela I., 2001, A\&A, 377, 161

Okazaki A. T., Bate M. R., Ogilvie G. I., Pringle J. E., 2002, MNRAS, 337, 967

Okazaki A. T., Hayasaki K., Moritani Y., 2013, PASJ, 65, 41

Papaloizou J. C. B., Lin D. N. C., 1994, in Duschl W. J., Frank J., Meyer F., Meyer-Hofmeister E., Tscharnuter W. M., eds, NATO ASI Series Vol. 417, Theory of Accretion Disks - 2. Kluwer Academic Publishers, Dordrecht, Netherlands, p. 329

Papaloizou J. C. B., Terquem C., 1995, MNRAS, 274, 987

Parkes G. E., Murdin P. G., Mason K. O., 1980, MNRAS, 190, 537

Paxton B., 2004, PASP, 116, 699

Postnov K. A., Mironov A. I., Lutovinov A. A., Shakura N. I., Kochetkova A. Y., Tsygankov S. S., 2015, MNRAS, 446, 1013

Pottschmidt K., Rothschild R. E., Gasaway T., Suchy S., Coburn W., 2006, BAAS, 38, 384

Priedhorsky W. C., Terrell J., 1983, ApJ, 273, 709

Rappaport S., Clark G. W., Cominsky L., Li F., Joss P. C., 1978, ApJ, 224, L1

Reig P., Fabregat J., Coe M. J., 1997, A\&A, 322, 193

Reig P., Larionov V., Negueruela I., Arkharov A. A., Kudryavtseva N. A., 2007, A\&A, 462, 1081

Rivinius T., Carciofi A. C., Martayan C., 2013, A\&ARv, 21, 69

Rothschild R. E., et al., 1998, ApJ, 496, 538

Rothschild R. E., et al., 2017, MNRAS, 466, 2752

Sugizaki M., Yamamoto T., Mihara T., Nakajima M., Makishima K., 2015, PASJ, 67, 73

Torres G., Andersen J., Giménez A., 2010, A\&ARv, 18, 67

Verner D. A., Ferland G. J., Korista K. T., Yakovlev D. G., 1996, ApJ, 465, 487

Walter K., 1975, A\&A, 42, 135

Wilms J., Allen A., McCray R., 2000, ApJ, 542, 914

Wisniewski J. P., Draper Z. H., Bjorkman K. S., Meade M. R., Bjorkman J. E., Kowalski A. F., 2010, ApJ, 709, 1306

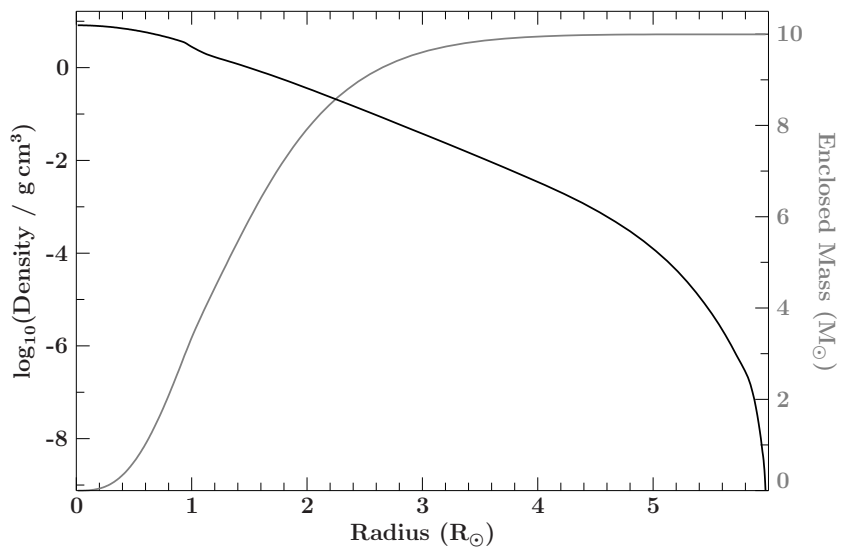

Figure A1. Used density profile (black) and enclosed mass (gray) of a star as a function of its stellar radius as calculated with the EZ code.

Yamamoto T., Sugizaki M., Mihara T., Nakajima M., Yamaoka K., Matsuoka M., Morii M., Makishima K., 2011, PASJ, 63, S751

Zamanov R. K., Reig P., Martí J., Coe M. J., Fabregat J., Tomov N. A., Valchev T., 2001, A\&A, 367, 884

This paper has been typeset from a $\mathrm{T}_{\mathrm{E}} \mathrm{X} / \mathrm{L} \mathrm{AT} \mathrm{E} \mathrm{X}$ file prepared by the author.

\section{APPENDIX A: STELLAR PRECESSION IN BINARIES}

In BeXRBs, if the rotation axis of the Be star is inclined with respect to the orbital plane, the compact object will exert a tidal torque on the Be star and disk. The torque on the Be star will be non-zero if the Be star is oblate (due to, e.g., fast rotation). In the following we estimate the resulting precession period of the Be star using two formulations by Kopal (1959, hereafter K59) and by Alexander (1976, hereafter A76). For all calculations we assume the orbital parameters as listed in Table 1, a stellar mass and radius for the $\mathrm{Be}$ star of $M_{*}=10 \mathrm{M}_{\odot}$ and $R_{*}=6 \mathrm{R}_{\odot}$, respectively (taken from Table 2), and a neutron star mass of $M_{X}=1.4 \mathrm{M}_{\odot}$. The radial density profile of the Be star as shown in Fig. A1 was calculated using the Evolve ZAMS (EZ) code ${ }^{3}$ (Paxton 2004) for an initial stellar mass and metallicity of $10 \mathrm{M}_{\odot}$ and 0.02 , respectively. We simulated stellar evolution up to an age of $15.6 \mathrm{Myr}$ in order to let the star expand up to the assumed radius of $6 \mathrm{R}_{\odot}$. When discussing other stellar radii or masses as shown in Fig. A2, we have linearly scaled the density profile for simplicity. In case of a different stellar mass, we scaled the density such that the total enclosed mass, i.e., the integral of the density profile over the full radius results in the assumed mass.

\section{A1 Formulation by K59}

Assuming that the inclination of the rotation axis of the primary star to the binary orbit axis is small, K59 derived the period of stellar precession as (K59, Eq. II.5-62)

$\frac{P_{\text {orb }}}{U_{1}}=\Gamma_{1}+\Pi_{1}$,

where $P_{\text {orb }}$ is the orbital period, $U_{1}$ is the period of precession of the primary star, and $\Gamma_{1}$ and $\Pi_{1}$ are quantities defined by (K59, Eq. II.5-25)

$\Gamma_{1}=\frac{3}{2} \frac{C_{1}^{\prime \prime}}{C_{1}^{\prime}} \gamma_{1}$

3 We have used the online tool EZ-Web developed by Richard Townsend, http://www.astro.wisc.edu/ townsend/static.php? ref=ez-web. 


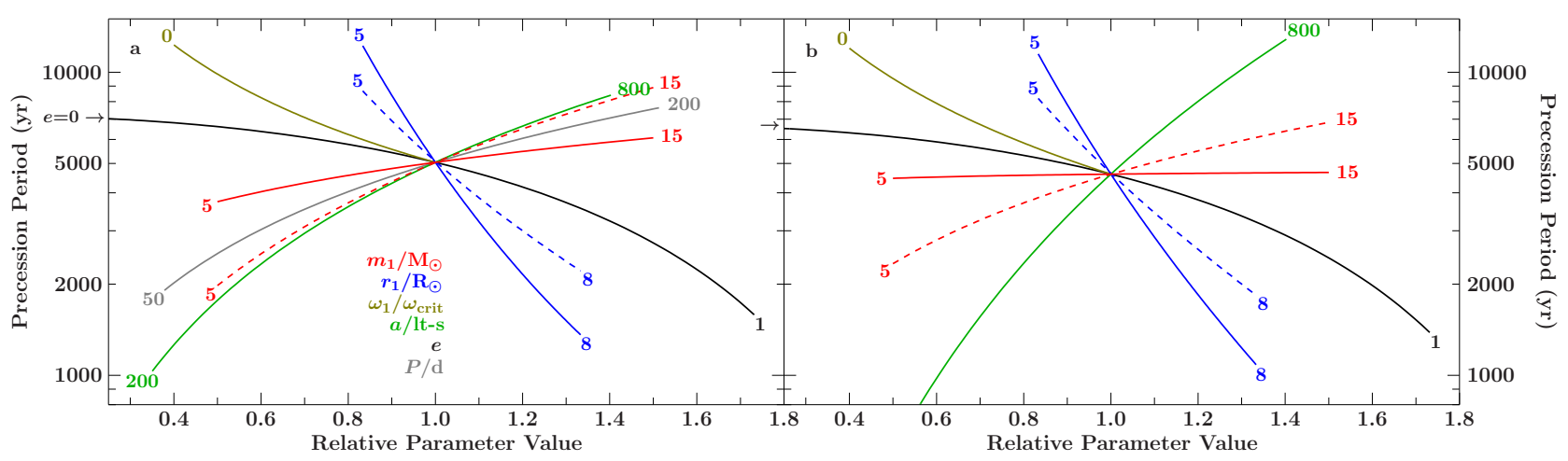

Figure A2. Dependencies of the precession period on stellar and binary parameters after a) K59 and b) A76. The parameter values are normalized to the ones assumed for GX 304-1 as given in the text. The parameter ranges are provided by absolute values at the line endings. The dashed lines have been calculated taking a linearly scaled density profile into account (see Fig. A1 and the text for details). The arrow on the y-axis labelled $e=0$ marks the value of the precession period for a circular orbit, to which the corresponding line (black) converges.

and (K59, Eq. II.5-33)

$\Pi_{1}=\frac{3}{4 a^{2}} \frac{C_{1}^{\prime}-A_{1}^{\prime}}{m_{1}}$.

Here, $m_{1}$ is the mass of the primary, $a$ is the semi-major axis of the binary, and $\gamma_{1}$ is the ratio of the rotation frequency of the primary, $\omega_{1}$, to the binary orbital frequency (K59, Eq. II.5-11),

$\gamma_{1}=\frac{\omega_{1}}{n}=\omega_{1} \sqrt{\frac{a^{3}}{G\left(m_{1}+m_{2}\right)}}$,

with the mass, $m_{2}$, of the secondary and the gravitational constant, $G$. The moment of inertia of the primary perpendicular, $A_{1}^{\prime}$, and parallel, $C_{1}^{\prime}$, to its rotational axis are given by (K59, Eq. II.3-34)

$A_{1}^{\prime}=\frac{8}{3} \pi \int_{0}^{r_{1}} \rho r^{\prime 4} \mathrm{~d} r^{\prime}-\frac{\left(\Delta_{2}-1\right) \omega_{1}^{2} r_{1}^{5}}{9 G}$

and by (K59, Eq. II.3-39)

$C_{1}^{\prime}=\frac{8}{3} \pi \int_{0}^{r_{1}} \rho r^{\prime 4} \mathrm{~d} r^{\prime}+\frac{2\left(\Delta_{2}-1\right) \omega_{1}^{2} r_{1}^{5}}{9 G}$,

respectively. Here, $\rho$ is the density of the primary star as a function of the distance, $r^{\prime}$, to its centre up to the full radius, $r_{1}$. In each equation, the first term on the right hand side is the moment of inertia of the primary at rest, which is calculated for the stellar structure as shown in Fig. A1. The second term describes the contribution of the rotational deformation to the moment of inertia, where $\Delta_{2}$ is related to the apsidal constant, $k_{2}$, as

$\Delta_{2}=1+k_{2}$.

We assume $k_{2}=0.01$ given that the apsidal constant of $10 \mathrm{M}_{\odot}$ mainsequence stars are typically in the range of $(5-8) \times 10^{-3}$ (Torres et al. 2010).

The tidal bulge forming inside the primary star due to the gravitational pull of the secondary modifies the moment of inertia. In the rotating reference frame of the orbital plane, this moment of inertia, $C_{1}^{\prime \prime}$, is calculated by (K59, Eq. II.3-46)

$C_{1}^{\prime \prime} \simeq \frac{1}{3}\left(\Delta_{2}-1\right) \frac{m_{2} r_{1}^{5}}{d^{3}}$

where $d$ is the distance of the secondary from the primary, which is given by

$d=\frac{a\left(1-e^{2}\right)}{1+e \cos f}$,

with $e$ and $f$ being the eccentricity and the true anomaly. When orbit averaged, Eq. A8 is reduced to

$C_{1}^{\prime \prime} \simeq \frac{\left(\Delta_{2}-1\right) m_{2} r_{1}^{5}}{3 a^{3}\left(1-e^{2}\right)^{3 / 2}}$

Using Eqs. A4-A6, the orbit average of $C_{1}^{\prime \prime}$ can also be written as

$C_{1}^{\prime \prime} \simeq\left(C_{1}^{\prime}-A_{1}^{\prime}\right) \frac{G m_{2}}{\omega_{1}^{2} a^{3}\left(1-e^{2}\right)^{3 / 2}}=\frac{m_{2}}{m_{1}+m_{2}} \frac{\left(C_{1}^{\prime}-A_{1}^{\prime}\right)}{\left(1-e^{2}\right)^{3 / 2} \gamma_{1}^{2}}$.
Note that the factor $\left(1-e^{2}\right)^{3 / 2}$ is set to 1 in K59, where the orbit is assumed to be almost circular. We retain this factor in Eq. A11 since the orbits of BeXRBs are generally eccentric.

With Eq. A11, we can write Eq. A2 as

$\Gamma_{1}=\frac{3}{2} \frac{C_{1}^{\prime \prime}}{C_{1}^{\prime}} \gamma_{1}=\frac{3}{2} \frac{m_{2}}{m_{1}+m_{2}} \frac{C_{1}^{\prime}-A_{1}^{\prime}}{C_{1}^{\prime}}\left(1-e^{2}\right)^{-3 / 2} \gamma_{1}^{-1}$.

Note that the power of $\gamma_{1}$ in Eq. A12 of -1 is different than in the original Eq. II.5-25 in K59 of +1, which has been noticed by, e.g., Walter (1975) already.

Assuming that the Be star is rotating at the critical speed, $\omega_{\text {crit }}$, as an upper limit,

$\omega_{1}=\omega_{\text {crit }}=\sqrt{G m_{1} / r_{1}^{3}}$,

and setting $r_{1}=R_{*}, m_{1}=M_{*}$, and $m_{2}=M_{\mathrm{x}}$ we finally find $U_{1} \sim 5000 \mathrm{yr}$ for the precession period of the Be star in GX 304-1 as calculated after Eq. A1. The dependencies of Eq. A1 on the stellar and binary parameters is shown in Fig. A2a.

\section{A2 Formulation by A76}

Alexander (1976) generalized the formulation of Kopal (1959) to an arbitrary angle, $\Theta$, between the angular momentum vectors of the primary and the binary orbit. If the angular momentum vectors of the Be star and its disk align, $\Theta$ is then equal to the disk misalignment angle, $\delta$, introduced in Sect. 4.2. In comparison to Eq. A1, Alexander (1976) derived (A76, Eq. 2.21)

$-\mu_{1} h_{1} \cos \Theta \frac{H}{H_{0}}=\frac{2 \pi}{U_{1}}$,

for the precession period, $U_{1}$, of the primary, which is negative for retrograde precession. Here, $\mu_{1}$ is a constant given by (A76, Eq. 2.18)

$\mu_{1}=\frac{1}{C_{1}+2 C_{\mathrm{R} 1}-C_{\mathrm{T} 1}} \frac{3 C_{\mathrm{T} 1}}{C_{1}+3 C_{\mathrm{T} 1}}$,

with the moment of inertia, $C_{1}$, of the primary star at rest, which is calculated by (A76, Eq. 2.4)

$C_{1}=\frac{8}{3} \pi \int_{0}^{r_{1}} \rho r^{\prime 4} \mathrm{~d} r^{\prime}$

and equal to the first term on the right hand side in Eq. A6. The rotational and tidal distortions to the total moment of inertia are are given by (A76, Eq. 2.5)

$C_{\mathrm{R} 1}=\frac{k_{2} \omega_{1}^{2} r_{1}^{5}}{9 G}$

and (A76, Eq. 2.14)

$C_{\mathrm{T} 1}=\frac{1}{6} k_{2} \frac{m_{2} r_{1}^{5}}{a^{3}\left(1-e^{2}\right)^{3 / 2}}$, 
respectively.

The total angular momentum, $H$, of the binary is defined as (A76, Eq. 2.19)

$\vec{h}_{1}+\vec{h}_{2}+H_{0} \vec{s}_{0}=\vec{H}$.

The angular momentum of the primary, $\vec{h}_{1}$, is expressed as (A76, Eq. 2.12)

$\vec{h}_{1}=\left(C_{1}+2 C_{\mathrm{R} 1}-C_{\mathrm{T} 1}\right) \vec{\omega}_{1}+3 C_{\mathrm{T} 1}\left(\vec{\omega}_{1} \cdot \vec{s}_{0}\right) \vec{s}_{0}$,

with its angular velocity vector, $\vec{\omega}_{1}$, and the normal vector, $\vec{s}_{0}$, of the orbital plane. Since the secondary is a compact object, which is a point mass in good approximation, its angular momentum is $\vec{h}_{2}=\overrightarrow{0}$. The orbital angular momentum of the binary, $H_{0}$, is given by (A76, Eq. 2.11)

$\vec{H}_{0}=H_{0} \vec{s}_{0}=\frac{m_{1} m_{2}}{m_{1}+m_{2}} \sqrt{G\left(m_{1}+m_{2}\right) a\left(1-e^{2}\right)} \vec{s}_{0}$,

Assuming a misalignment angle $\Theta=0$ as an upper limit and the same parameters for GX 304-1 as previously in Sect. A1, we find a precession period of $U_{1} \sim-4600 \mathrm{yr}$ using Eq. A14, which agrees with the estimation following K59. Fig. A2b shows the dependencies of Eq. A14 on the stellar and binary parameters.

\section{A3 Parameter dependencies}

The precession period of the stellar companion star in GX 304-1, which we derived following K59 and A76, is around $5000 \mathrm{yr}$ and both formulations agree within a few hundred years. This period does not explain the short period of $\sim 2 \mathrm{yr}$ we have discovered by modelling the evolution of the absorption column density (see Sect. 4), which we interpret as the precession period of the Be disk.

Since we have fixed the stellar and binary parameters for the estimation of the stellar precession period to the values given above, different parameter values might change the resulting period significantly. Figure A2 shows the dependency of the stellar precession period after K59 and A76 on these parameters within a reasonable range. As can be seen, the precession period is larger than $1000 \mathrm{yr}$ in almost all cases. Thus, it is unlikely that a certain combination of parameters indeed results in a precession period consistent with our findings.

We note that there is a difference between the formulations by K59 and A76, which is most prominent in the dependency on the primary mass, $m_{1}$ (red curve in Fig. A2), and the semi-major axis, $a$ (green curve). This is due to the fact that A76 uses Kepler's third law,

$\frac{P_{\text {orb }}^{2}}{a^{3}}=\frac{4 \pi^{2}}{G\left(m_{1}+m_{2}\right)}$,

to calculate the binary orbital period, $P_{\text {orb }}$, while the equations in K59 have a direct dependency. Indeed, when substituting $P_{\text {orb }}$ in Eq. A1 with Eq. A22, both formulations agree very well. 